\title{
SELFADJOINT OPERATOR EXTENSIONS SATISFYING THE WEYL COMMUTATION RELATIONS
}

\author{
BY PALLE E. T. J $\varnothing$ RGENSEN 1
}

\begin{abstract}
Motivated by questions concerning uniqueness of unbounded derivations in commutative $C^{*}$-algebras, and related problems on singular perturbations, we define two mixed global and infinitesimal versions of the Weyl operator commutation relations (one degree of freedom and infinite multiplicity), a weak one and a strong one. We announce two structure theorems of a geometric nature which characterize the nonselfadjoint symmetric operators entering in the Weyl systems. Proofs are only indicated.
\end{abstract}

Our starting point is the following variant of the Stone-von Neumann Uniqueness Theorem [12], [4b]. Let $(U, V)$ be a pair of unitary one-parameter groups (always assumed strongly continuous) of operators on a separable Hilbert space $H$, and suppose that the Weyl commutation relation

$$
U(t) V(s)=V(s) U(t) e^{i t s} \quad(\text { for all } s, t \in \mathbf{R})
$$

holds. Then it is possible to represent the system in the form $S U(t) S^{-1} f(x)=$ $f(x+t), S V(s) S^{-1} f(x)=e^{i s x} f(x)$, where $S$ is an isometry of a space $L^{2}(\mathbf{R}, N)$ of the norm-square integrable functions $f$, with values in a separable Hilbert space $N$, onto $H$; the dimension of $N$ being equal to the (uniform) multiplicity of the spectrum of $U .^{2}$

Instead of (1) we consider the following infinitesimal Weyl relation with symmetric but generally nonselfadjoint generator. Let $\{U(t)\}_{t \in R}$ be a unitary one-parameter group on $H$, and let $Q$ be a symmetric operator with dense domain $D(Q)$ in $H$. The corresponding relation

$$
(U(t) Q f, g)=(U(t) f, Q g)+t(U(t) f, g) \quad \text { for all } f, g \in D(Q)
$$

is here called the infinitesimal Weyl relation for the triple $(U, Q, H)$. It is clearly equivalent to (1) if $Q$ is essentially selfadjoint. But in scattering theory of singular perturbations, and in recent investigations of the author concerning uniqueness of unbounded derivations, the relation (2) for nonselfadjoint $Q$ plays an interesting role. Simple examples show that the operator $Q$ of a given system $(U, Q, H)$ may

Received by the editors August 3, 1978.

AMS (MOS) subject classifications (1970). Primary 47B25, 81 A20; Secondary 43A65, $46 \mathrm{~K} 99,47 \mathrm{~B} 47$.

Key words and phrases. Weyl relations, operator domains, unitary groups.

1 Research supported in part by NSF Grant MCS 77-02831 and sequel.

${ }^{2}$ We refer to [LP] as a general reference, containing in addition important applications. 
have nonzero defect indices [4a] which can be equal or unequal. If they are equal, it may or may not be possible to extend $Q$ to a selfadjoint operator $\widetilde{Q}$ so that the one-parameter group $V(s)=e^{i s \widetilde{Q}}$ is part of a (global) Weyl system $(U, V)$, i.e. such that (1) holds. The triples $(U, Q, H)$ which can be extended to global Weyl systems are called extendable.

In view of the negative examples alluded to above, it may be surprising that a certain canonical and minimal extension always exists.

THEOREM 1. Let $(U, Q, H)$ be an infinitesimal Weyl system. Then symmetric extensions $Q_{1}$ of $Q$ exist such that

$$
D\left(Q_{1}\right) \text { is invariant under } U(t) \text { for all } t \in \mathbf{R} \text {, and }
$$

$$
Q_{1} U(t) f=U(t) Q_{1} f-t U(t) f \text { for all } f \in D\left(Q_{1}\right) \text {. }
$$

There is a unique smallest symmetric extension $Q_{m}$ satisfying (3), i.e. $Q_{m} \subseteq Q_{1}$ for all symmetric extensions $Q_{1}$ satisfying (3). The operator closure $\bar{Q}_{m}$ satisfies (3) as well and is the unique smallest symmetric and closed extension of $Q$ satisfying (3).

Here is an easy

Corollary 2. If the system $(U, Q, H)$ is extendable to a global Weyl system $(U, V)$ with $V(s)=e^{i s \widetilde{Q}}$ then $Q_{m} \subseteq \widetilde{Q}$.

Definition. We say that the system $(U, Q, H)$ has $U$-indices $(p, q)$ if the minimal operator $Q_{m}$ has defect indices $(p, q)$. It can readily be shown that the $U$-indices are equal whenever $Q$ has equal defect indices, but in general the indices of $Q$ may be infinite while those of $Q_{m}$ are finite. Theorem 4 below is a converse to Corollary 2.

TheOREM 3. Let $(U, Q, H)$ be an infinitesimal Weyl system with at least one finite $U$-index. Then there is a Hilbert space $K$ containing $H$, and a global Weyl system $(U, V)$ on $H$ such that $U$ reduces to $U$ on $H, H$ is semi-invariant [9] for $V$, and $Q$ is contained in the infinitesimal generator for the contraction semigroup $\left.s \rightarrow P_{H} V(s)\right|_{H}$.

A structure theory for the associated contraction semigroups, due to $\mathbf{P}$. Muhly, will appear in a joint article with the author [2b].

The next result concerns the relation (3) for extendable systems. Hence it is stated for operators in $L^{2}$-spaces, and $U(t)$ is translation. Vanishing conditions of the Fourier transform on a set of measure zero clearly give rise to systems (3). The theorem is a partial converse to this statement.

THEOREM 4. Let $Q_{1}$ be a symmetric and closed operator in $L^{2}(\mathbf{R})$ which is contained in the multiplication operator $\widetilde{Q} h(x)=x h(x)$. Assume that $D\left(Q_{1}\right)$ is translation invariant, and that $D\left(\widetilde{Q} Q_{1}\right)$ is a core for $Q_{1}$. Then (3) holds and 
the closed set $\Lambda=\left\{\lambda \in \mathbf{R} \mid \hat{h}(\lambda)=0\right.$ for $\left.\forall h \in D\left(Q_{1}\right)\right\}$ is of zero measure. Moreover $D\left(Q_{1}\right)=\{h \in D(\widetilde{Q}) \mid \hat{h}(\lambda)=0$ for $\forall \lambda \in \Lambda\}$.

The core condition can be omitted in the following cases: (1) $\Lambda$ is a Cantor set, or (2) $Q_{1}$ has finite defect. In general it can be slightly weakened, but not omitted. In case (2) the result generalizes to arbitrary multiplicity.

The proofs of results 1 through 3 involve general operator theory [1], including theorems of Phillips [5] and Naimark [3], while the proof of Theorem 4 is based on a function theoretic approach to the extension theory of [4a] made possible by the extendability assumption and the Stone-von Neumann Theorem. (The function theory is based on Wiener-Tauberian consideration.)

Generalizations to group representations [2a], [6], [7], [8], [11] and field theory [10] would be of interest. The possibility of removing the finiteness assumption in Theorem 3 is related via the proof to a well-known conjecture of Phillips [5]. We conjecture the conclusion of Theorem 4, also without the finiteness assumption on the $U$-index, but it seems hard to settle either of the conjectures. It appears difficult in the general case, for two different extensions to establish the existence of a wave operator similarity which commutes with $U$.

However the extendability question has an answer in full generality, i.e. no restriction on the indices. The proof uses arguments that naturally extend [4a].

Theorem 5. Let $(U, Q, H)$ be an infinitesimal Weyl system, and let $\boldsymbol{P}_{ \pm}$ denote the orthogonal projections onto the respective defect spaces $D_{ \pm}$for $Q_{m}$. The system is extendable if and only if there exists a partial isometry $S$ of $D_{+}$ onto $D_{-}$such that $P_{-}(2[S, U(t)]-i t(I+S) U(t)(I+S)) P_{+}=0$ for all $t \in \mathbf{R}$. Here $[\cdot, \cdot]$ denotes the commutator bracket.

If it is assumed in addition that the spectral measure $d E_{\lambda}$ of $U$ is absolutely continuous, then extendability is equivalent to the validity of the following identity

$$
P_{-}\left(2\left[S, E_{\lambda}\right]-(I+S) D_{\lambda}(I+S)\right) P_{+}=0 \quad(\lambda \in \mathbf{R})
$$

for some partial isometry $S$ of $D_{+}$onto $D_{-}$. Here $D_{\lambda}$ denotes the Radon-Nikodym derivative of $d E_{\lambda}$.

We finally point out that Theorem 5 has a complete generalization to the case when the $W^{*}$ algebra generated by the spectral projections of $U$ is replaced by an arbitrary noncommutative $W^{*}$-algebra, and when the map $U(t) \rightarrow$ it $U(t)$ is replaced by a spatial derivation which is implemented by a symmetric nonselfadjoint operator. This answers a question raised in a recent article of the author.

The author has benefitted greatly from discussions with Professors P. Chernoff, W. Helton, G. Johnson, R. T. Moore, P. Muhly, and R. S. Phillips. I am pleased to record my gratitude.

\section{REFERENCES}

1. C. Foiaş and L. Gehér, Über die Weylsche Vertauschungsrelationen, Acta Sci. Math. 24 (1963), 97-102. 
2. P. E. T. Jørgensen (a) (with R. T. Moore), Commutation relations for operators, semigroups, and resolvents in mathematical physics and group representations (preprint); (b) (with P. S. Muhly), Selfadjoint extensions satisfying the Weyl operator commutation relations (in preparation).

3. M. A. Naìmark, On commuting unitary operators in spaces with indefinite metric, Acta Sci. Math. 24 (1963), 177-189.

4. J. von Neumann, (a) Allgemeine Eigenwerttheorie Hermitescher Funktionaloperatoren, Math. Ann. 102 (1929-30) 49-131; (b) Die Eindentigkeit der Schrödingerschen Operatoren, Math. Ann. 104 (1931), 570-578.

5. R. S. Phillips, On dissipative operators, Lecture Series in Differential Equations, vol. II, A. K. Aziz (Ed.), Van Nostrand Mathematical Studies no. 19, Van Nostrand, New York, 1969.

6. N. S. Poulsen, On $C^{\infty}$ vectors and interwining bilinear forms for representations of Lie groups, J. Functional Analysis, (1972), 87-120.

7. R. T. Powers, On selfadjoint algebras of unbounded operators, Trans. Amer. Math. Soc. 187 (1974), 261-293.

8. R. R. Rao, Unitary representations defined by boundary conditions-the case of sl(2, R), Acta Math. 139 (1977), 185-216.

9. D. Sarason, On spectral sets having connected complement, Acta Sci. Math. 26 (1965), 289-299.

10. I. E. Segal, Nonlinear functions of weak processes. II, J. Functional Analysis 6 (1970), 29-75.

11. I. M. Singer, Lie algebras of unbounded operators, Thesis, Univ. of Chicago, 1950.

12. M. H. Stone, Linear transformations in Hilbert space. III. Operational methods and group theory, Proc. Nat. Acad. Sci. U.S.A. 16 (1930), 172-175.

[LP] P. D. Lax and R. S. Phillips, Scattering theory, Academic Press, New York, 1967.

DEPARTMENT OF MATHEMATICS, STANFORD UNIVERSITY, STANFORD, CALIFORNIA 94305 